

\section{Time Evolution of Mass Flows \\ in a Collisional Tokamak}

by

Adil B. Hassam and Russell M. Kulsrud

Princeton University, Plasma Physics Laboratory

Princeton, New Jersey 08540 USA

\section{ABSTRACT}

The time evolution, due to dissipati re processes. of an initial pattern of poloidal and toroidal mass flows in a tokamak is considered. The calculation is applicable to a collisional, low $\beta$, axisymmetric tokam:k of arbitrary minor cross section. Time rates of change of poloidal flows which are subsonic but larger unan the diamagnetic speed are given according to the magnitude of the flow and the collisionality of the plasma. Over most of parameter space for typical tokamaks, the poloidal rotation is strongly damped by magnetic pumping at the rate $(\ell / q R)^{2} v_{i i}$, where $\ell$ is the mean free path, $q R$ the "connection length," and $v_{i i}$ the ion-ion collision frequency. At higher speeds, even stronger damping is effected by electron thermal conduction. The toroidal rotation is determined largely by the conservation of toroidal angular momentum. A heuristic explanation of the damping due to magnetic pumping is given. 


\section{INTRODUCTION}

Various processes tend to drive large poloidal and toroidal plasma flows in a tokamak. This tendency is counteracted by dissipative mechanisms within the plasma which serve to damp such flows. In this paper, we consider the manner in which ar initial flow pattern in a tokamak evolves in time as a result of dissipative effects within the plasma. Time rates of change of both poloidal and toroidal components are obtained.

The calculation is applicable to a collisional, lov $\beta$, axisymmetric tokamak plasma of general minor cross section. The magnetic field is included self consistently. We restrict our attention to flows whose magnitude is intermediate between the "poloidal sound speed" $\theta C_{S}$ and the diamagnetic speed $U, i, e$. $U<v<\theta C_{S}$. Further, we concern ourselves only with time scales shorter than the classical ion energy confinement time $(\mathrm{m} / \mathrm{M})^{1 / 2}{ }^{\mathrm{T}} \mathrm{ps}$ ( $\tau_{p_{s}}$ is the well known "Pfirsch-Schlijter time" and $m, M$ aize the electron, ion masses, respectively). Within the confines of the above limits, the available parameter space is systematically explored. Previously known results are recovered. (A review of previous work is given by Green ${ }^{l}$.)

After describing the character of a general flow pattern in a tokamak in Section II, we describe in some detail the results for the rate of change of poloidal and toroidal flows in section III. A heuristic derivation of the principal process damping poloidal rotation is given in section $I x$ at the end of the paper. The remaining sections, starting from Section IV, describe the mathematical analysis employed. 
II. THE FLOW PATTERN

A general flow pattern in a tokamak is best viewed as being composed of free flow of plasma along magnetic flux tubes, $v_{\|}$, superimposed on any "motion" of the flux tubes themselves (Fig. 1) (in analogy to a bead on a helical wire). The flux tube motion $v_{E}$ arises strictly in response to a radial electric field and constitutes an $\vec{E} \times \vec{B}$ rotation of the entire flux tube. In this view, a strictly toroidal flow, for example, is composed of both parailel flow and perpendicular flux tube rotation, although, in view of the fact that the ratio between the poloidal and toroidal field strengths $\theta \equiv \mathrm{B}_{\mathrm{p}} / \mathrm{B}_{\mathrm{T}}$ is ordinarily small, the former component is of larger magnitude. For subsonic speeds, a general flow pattern is kept largely incompressible by the uresence of the rotational transform.

In practice, parallel flows may be driven in a tokamak as a result of a transfer of mechanical momencum from toroidally injected neutral beams. Radial electric fields may arise as a consequence of several non ambipolar effects such as charged particle losses resulting from nuclear reactions, perpendicl'ar neutral beam injection, or R. F, heating. Electric fields may also arise spontaneously as a result of resistive diffusion as was shown by stringer. ${ }^{2}$ In the absence of driving forces and dissipative processes, a given flow pattern will persist indefinitely. Such a flow is characterized by two constants for each magnetic surface corresponding to the net amount of toroidal angular momentum on the surface and the electric potential of the surface. In this paper, we calculate the time evolution of these constants which results from dissipative processes; for simplicity, we assume the driving 
forces to be absent. The results are given in the next section.

III. SUMMARY OF RESULTS

The exact results for the time rates of change of the poloidal and toroidal flows in a tokamak with circular cross sections and small inverse aspect ratio are given by Eqs. (30) and (31). The results for more general cross sectional shapes differ from (30) and (31) by multiplicative factors of order one which may be deduced from Eqs. (21) - (29). In this section, we describe the results qualitatively.

\section{Poloidal Rotation}

The rate at which a given amount of poloidal rotation $v_{p}$ decays depends on the collisionality of the plasma and the magnitude of the poloidal speed. This is summarized schematically in Fig. 2 in which the collisionality is represented on the abscissa by $\ell / q R$, $\ell$ being the mean free path and $q R$ the usual tokamak "connection length", and the polojdal speed is plotted on the ordinate. With the exception of the short mean free path region (shaded), the poloidal rotation is strongly damped cver most of parameter space. The dominant damping mechanism is the parallel ion viscosity $\eta_{0}$ which damps the rotation at a rate $(\ell / q R)^{2} v_{i i}$, where $v_{i i}$ is the ion-ion collision time. This damping effect may be physically interpreted as resulting from a magnetic pumping of the plasma as the plasma rotates through the inhomogenous magnetic field (we give a heuristic derivation of this effect in section IX). The importance of parallel viscosity was pointed out by Rosenbluth et al.,$^{3}$ and the above damping 
rate has also been obtained by Boozer ${ }^{4}$ and by Chrisman et. al.$^{5}$ Magnetic pumping has been shown to be equally important in the collisionless regime $(q R<\ell)$ by stix. 6

At higher poloidal speeds (see Fig. 2 for more exact demarcations), effects due to parallel electron heat conductivity $k_{e}$ damp the rotation at an even faster rate. The rates differ depending on whether the parallel heat conduction time is shorter than the rotation time or vice versa. In the former case (corresponding to the region denoted by $\left.1 / k_{e}\right)$, the damping rate is of order $v_{i i}(m / M){ }^{1 / 2}\left(v_{p} / \theta C_{s}\right)^{4}$. In the latter case (denoted by $\mathrm{K}_{\mathrm{e}} \mathrm{j}$, the damping proceeds at a rate of order $v_{i i}(M / m)^{1 / 2}\left(v_{p} / \theta c_{s}\right)^{2}(l / q R)^{2}$. This damping due to parallel thermal conduction is physically akir to the damping due to magnetic pumping.

In the short mean free path region, (shaded), i.e., $(l / q R)<(\rho / \theta a)(m / M)^{1 / 4}$, the plasma is unstable to poloidal rotation ( $\rho$ is the ion gyroradius and a the density gradient scale length). This is the well-known stringer "poloidal spinup" 2 which is driven by a coupling of the poloidal rotation to the resistive "Pfirsch-schlüter" flow. The growth time ${ }^{7}$ for the spin-up is of order $\theta^{2} q^{2} \tau_{p s} \quad$ ( $q$ being the usual tokamak "safety factor"). If the mean free path in this resistive region is not too short, i.e. $(\mathrm{m} / \mathrm{M})^{1 / 2}(\rho / \theta a j<(l / q R)$, the spin up is arrested by the stabilizing effect of $k_{e}$ to give a stable subsonic rotation speed of order $\theta \mathrm{C}_{\mathrm{S}}(\rho / \theta \mathrm{a})(\mathrm{gR} / \mathrm{l})(\mathrm{m} / \mathrm{M})^{1 / 2}$. If the inequality is reversed, the spin up may proceed up to the poloidal sound speed. At this level, shocks may arise (described in Ref. 8) or perpendicular ion viscosity may stabilize the spin-up ldescribed 
in Ref. 9). (In fact, if $(\mathrm{m} / \mathrm{M})^{1 / 2}<\theta^{2}$, perpendicular viscosity prevents the spin-up for all $\mathrm{v}_{\mathrm{p}}{ }^{9}$. For the present paper, we assume $\theta^{2}<(m / M)^{1 / 2}$ and ignore perpendicular viscous effects).

For present day tokamaks, where $\ell$ - $q R$, the strong damping resulting from magnetic pumping should serve to make any finite poloidal rotation unlikely, at least at speeds much in excess of the diamagnetic speed. This seems to be borne out experimentally.10,11 Poroidal Rotation

In principle the total toroidal angular momentum of the plasma is always conserved. (In practice, damping due to neutrals, not included herein, may be important. ${ }^{12}$, This is a consequence of the axisymmetry of the system. There may however be a redistribution of momentum from surface to surface due to (a) convection from resistive diffusion at a rate $\tau^{\tau s}{ }^{-1}$, (b) a coupling of the Pfirsch-schlïter flow with the poloidal flow, at a rate $\left(\theta_{\mathrm{ps}}\right)^{-1}$, or (c) the effects of perpendicular viscosity which may be expected to iron out shear flows at a rate comparable to the ion energy confinement rate (see Ref. 3). IV. EQUATIONS AND METHOD

In the collisional regime, $\ell<q R$, it is appropriate to use the plasma transport equations as given by Rraginskii. 13 For our purposes, it suffices to use a simplified version of these equations as given by:

$$
a n / \partial t+\vec{\nabla} \cdot n \vec{v}=0
$$




$$
\begin{aligned}
& n M(\partial \vec{v} / \partial t+\vec{v} \cdot \vec{\nabla} \vec{v})=-\vec{\nabla} p-\vec{\nabla} \cdot \vec{\Pi}+\vec{j} \times \vec{B} \\
& \overleftrightarrow{\Pi} \equiv-3 n_{0}(\hat{\mathrm{bb}}-\overleftrightarrow{I} / 3)(\hat{\mathrm{b}} \hat{\mathrm{b}}-\overleftrightarrow{I} / 3): \vec{\nabla} \vec{v} \\
& p \equiv n\left(T_{e}+T_{i}\right) \\
& n T(\partial s / \partial t+\vec{v} \cdot \vec{\nabla} s)=-\vec{\nabla} \cdot \vec{q}-\overleftrightarrow{\Pi}: \vec{\nabla} \vec{v} \\
& s \equiv(3 / 2) \ln \left(T / n^{2 / 3}\right) \\
& \overrightarrow{\mathrm{q}} \equiv-k \mathrm{~B}^{-2} \overrightarrow{\mathrm{B} B} \cdot \vec{\nabla} \mathrm{T} \\
& \vec{E}=-\vec{v} \times \vec{B}+\eta \vec{j} \\
& \vec{\nabla} \times \vec{B}=\vec{j} \\
& \vec{\nabla} \times \vec{E}=0 \quad \vec{\nabla} \cdot \vec{B}=0
\end{aligned}
$$

Standard notation is employed with $\hat{b} \equiv \vec{B} /|\vec{B}|$. Equations (3) are understood to apply either to elisctrons or to ions. $s_{e}$ and $s_{i}$ are the respective entropies, $k$ is the appropriate parallel thermal conductivity as defined in Ref. 13 , and $\vec{q}$ is the parallel heat flux. Equation (2b) defines the stress tensor, $\overleftrightarrow{\Pi}$, where $n_{0} \equiv 96 \mathrm{nT}_{i} / \nu_{i i}$ is the parallel ion viscous coefficient. This form $^{14}$ for $\overleftrightarrow{\Pi}$ preserves toroidal angular momentum for an axisymmetric toroid, as it should. 
The neglect of various terms in the above version of the transport equations may be shown to be self consistent with the restrictions set forth in the intrcduction. Such terms include the gyroviscosity, 9 "drift" and perpendicular heat conductivity, 15 and additional terms in $\overleftrightarrow{\Pi}$ arising from heat flow. ${ }^{16}$ careful consideration shows that the thermal force and the related heat flux ${ }^{\mathrm{J} .7}$ may also be neglected. Further, in neglecting any time variation of $\vec{B}$, we have assumed the field to be totally relaxed with respect to the skin effect, and that any other time variations occur on confinement time scales. 18

Our mathematical treatment of the time evolution of Ilows in axisymetric equilibrium proceeds by a series of approximations as follows. Since the flows are subsonic, we first start from the usual static axisymmetric equilibrium. Next, we allow a mass flow which is a solution of the time independent ideal MHD equations (Section $V$ ). In the absence of dissipation, these flows persist indefinitely. Finally, in the last approximation, the dissipative effects are introduced. This approximation is justified only if $l<q R$. The introduction of dissipation in this perturbative manner implies consistency conditions on the lower order equilibrium (Section VI). These conditions yield the time evolution of the flow. An inverse aspect ratio expansion is utilized in Section VII to simplify the equations. Exact time rates of change are obtained for circular cross sections in Section VIII. 


\section{EQUILIBRIUM}

The equations describing the equilibrium are obtained from equations (1)-(5) by setting all time derivatives to zero and, in all but the heat equations, neglecting all dissipative terms. As far as the heat equation for each species is concerned, there are two limiting situations which give ideal MHD equations. If the paraliel heat flow is small, the species rotates adiabatically and the heat flow may be treated as a dissipative correction. This occurs if the poloidal rotation rate $\left(v_{p} / a\right)$ is large compared to the rate of thermal relaxation along the magnetic field lines $v(\ell / q R)^{2}$; (which implies, say for ions, the inequality $\left.\ell / q R<v_{p} / \theta C_{s}\right)$. In this case one has, in lowest order, $\vec{v} \cdot \vec{\nabla} s=0$. In the opposite limit in which the parallel heat flow is dominant, one has isothermal. flow, and departures from isothermality may then be treated as corrections. The lowest order equation is then $\vec{\nabla} \cdot \vec{q}=0$. It is clearly possible for the electrons to be nearly isothermal and the ions nearly adiabatic. These limiting cases divide the parameter space introduced in Fig. 2 into three physically distinct regions corresponding to an adiabatic regime $v_{p} / \theta C_{s}>(M / m)^{1 / 2} \ell / q R$, an intermediate "mixed" regime, and an isothermal regime $v_{p} / \theta C_{s}<\ell / q R$. In the subsequent analysis, we solve the equations separately for each region.

The equilibrium equations, obtained as just described, are first solved to lowest order in $\mathrm{v} / \theta \mathrm{C}_{\mathrm{S}}$. Inertial corrections, entering from the $n M \vec{v} \cdot \vec{\nabla} \vec{v}$ term, are obtained later as needed. We consider nested magnetic surfaces for simplicity. Following Ref. 7 we define an axisymmetric orthogonal coordinate system $(r, \theta, z)$ (Fig. 3) where $r$ labels a magnetic surface, $z$ is the 
aximuthal coordinate, and $\theta$ is a generalized poloidal angular coordinate whose constant surfaces and orthogonal to constant $r$ and $z$ surfaces. The coordinates $r$ and $\theta$ may be chosen arbitrarily although we imagine them to be close to polar coordinates. The line element squared is written $\mathrm{ds}^{2}=\mathrm{h}_{I}^{2} \mathrm{dr}{ }^{2}+\mathrm{h}_{\theta}^{2} \mathrm{d \theta}{ }^{2}+\mathrm{h}^{2} \mathrm{dz} \mathrm{z}^{2}$. (For circular concentric cross sections, for example, $h_{Y}=1$, $h_{\theta}=r, h=1+(r / R) \cos \theta$, where $R$ is the distance from the center line to the magnetic axis.) 'ro lowest order in $v / \mathrm{OC}_{\mathbf{s}}$, the equilibrium equations are simply the usual magnetostatic equations. The solutions are well-known. ${ }^{7}$ The magnetic field is written

$$
\vec{B}=\left(0, \theta(r) / h_{r}, 1\right) B_{0}(r) / h
$$

where $B o(r)$ and $\theta(r)$ are surface functions which are related to each other according to the Grad Shafranov equation

$$
\left(h^{2} \theta B_{0} / J\right)\left(h_{\theta}^{2} \Theta B_{0} / J\right)^{\prime}=-B_{0} B_{0}^{\prime}-h^{2} p^{\prime}
$$

where $J \equiv h_{r} h_{\theta} h$ and primes denote differentiation with respect to $r$. The pressure $\mathrm{p}$ is also a surface function. The radiri current is zero while the poloidal and perpendicular components of the current are, respectively,

$$
j_{\theta}=-B_{0}^{\prime} / h_{r}, j_{\perp}=\left(p^{\prime} / B_{0}\right) h b_{z} / h_{r} \text {. }
$$

where $b_{z}^{-2} \equiv 1+\theta^{2} / h_{r}^{2}$. The poloidal component $j_{\theta}$ is the projection of $\vec{j}$ onto the $\hat{\theta}$ airection, while the perpendicular component $j_{1}$ is the projection in a direction perpendicular to $\vec{B}$ and ? ving on the surface. In terms of these, the toroidal component $j_{z}=\vec{j} \cdot \vec{z}$ and the parallel component $j_{\|}=\vec{j} \cdot \vec{b}$ are given by 


$$
\begin{gathered}
\theta j_{z}=-B_{0}^{\prime} / h-\left(p^{\prime} / B_{o}\right) i 1 \\
\theta j_{\|}=-B_{0} / / h b_{z}-\left(p^{\prime} / P_{o}\right) h b_{z}
\end{gathered}
$$

The flows may now be written down. The curl free electric field is written most generally as

$$
\vec{E}=-\vec{\nabla} \phi+(\hat{\varepsilon} / \mathrm{h}) \hat{z}
$$

where $\mathcal{E}=$ const. is the externally induced electric field. However, $\varepsilon$ scales with the resisivity $n$ and, to lowest order in $n$, we deduce from the parallel component of Ohm's Law (4) that $\phi=\phi(x)$. The incompressibility condition $\vec{\nabla} \cdot \vec{v}=0$ and Ohm's Law $\vec{v} \times \vec{B}=\vec{\nabla} \phi$ then, respectively, determine the poloidal and perpendicular components of $\vec{v} \underline{v i z}$.

$$
v_{\theta}=v_{p}(r) / h h_{r} \quad v_{\perp}=v_{E}(r) h b_{z} / h_{r}
$$

where $v_{p}(r)$ is a surface function and $v_{E}(r) \equiv \phi^{\prime} / B_{O}$. The parallel and toroidal projections of $\vec{v}$ are directly analogous to those of $\vec{j}$.

Finally, the appropriate heat equations for each species give the equations of state

$$
\begin{aligned}
& T=T(x) \text { for an isothermal species } \\
& s=s(x) \text { for an adiabatic species }
\end{aligned}
$$


The equilibrium is now characterized by six indeperdent surface functions which we take to be $B_{O}, p, v_{p}, v_{E}$ and a thermodynamic function for each species.

VI. CONSISTENCY CONDITIONS

Consistency conditions determining the slow time evolution of the zero order equilibrium quantities are obtained in the usual way by annihilating the leading order terms in the exact equations (1)-(5). Defining a flux surface average by $\langle E\rangle \equiv\left(\int \mathrm{dS} /|\vec{\nabla} \mathrm{p}|\right)^{-1} \int \mathrm{dSf} /|\vec{\nabla} \mathrm{p}|$, we find the consistency conditions to be:

$$
\begin{aligned}
& (\partial / \partial t)\langle n\rangle+\left(\int d \mathbf{s} /|\vec{\nabla} p|\right)^{-1}(\partial / \partial p) \int d \vec{s} \cdot n \vec{v}=0 \\
& (\partial / \partial t)\langle\vec{v} \cdot \vec{B}\rangle+\left(\int d s /\left|\vec{v}_{p}\right|\right)^{-1}(\partial / \partial p) \int d \vec{s} \cdot \vec{v} \vec{v} \cdot \vec{B} \\
& =-\vec{v} \cdot\langle\vec{\nabla} \times(n \vec{j}-\hat{z} \hat{E} / h)\rangle \\
& -\left\langle 3 \eta_{0}(n M)^{-1} \vec{B} \cdot \vec{\nabla} \ln B \vec{v} \cdot \vec{\nabla} \ln B\right\rangle \\
& +\mathrm{M}^{-1} \sum_{i, e}\langle\mathrm{~TB} \cdot \vec{\nabla} \mathrm{s}\rangle
\end{aligned}
$$

$(\partial / \partial t)\langle\mathrm{nMRh} \dot{v} \cdot \vec{z}\rangle+\left(\int \mathrm{dS} /|\vec{\nabla} \mathrm{p}|\right)^{-1}(\partial / \partial \mathrm{p}) \int \mathrm{d} \overrightarrow{\mathrm{S}} \cdot \overrightarrow{\mathrm{v}}(\mathrm{nMRh} \overrightarrow{\mathrm{v}} \cdot \vec{z})=0$ 


$$
\begin{aligned}
\langle n\rangle \partial \mathrm{s} / \partial t & +\left(\int \mathrm{as} /|\vec{\nabla} \mathrm{p}|\right)^{-1} s \overrightarrow{\mathrm{S}} \cdot \mathrm{nv}(\partial \mathrm{s} / \partial \mathrm{p}) \\
& \simeq\left\langle\kappa(\vec{B} \cdot \vec{\nabla} \mathrm{T} / \mathrm{BT})^{2}\right\rangle \\
& +\left\langle\left(3 n_{0} / \mathrm{T}\right)(\vec{v} \cdot \vec{\nabla} \ln B)^{2}\right\rangle
\end{aligned}
$$

$$
\begin{aligned}
\mathrm{T}\langle\mathrm{n} a \mathrm{~s} / \partial t\rangle & +\mathrm{T}\left(\int \mathrm{d} s /|\vec{\nabla} \mathrm{p}|\right)^{-1}(\partial \mathrm{s} / \partial \mathrm{p}) \int \mathrm{d} \overrightarrow{\mathrm{S}} \cdot \mathrm{nv} \\
& \simeq-\left(n v_{\theta} / B_{\theta}\right)\langle\mathrm{TB} \cdot \vec{\nabla} \mathrm{s}\rangle \\
& +\left\langle 3 n_{0}(\overrightarrow{\mathrm{v}} \cdot \vec{\nabla} \ell \mathrm{nB})^{2}\right\rangle
\end{aligned}
$$

$$
\eta\langle\vec{j} \cdot \vec{B}\rangle=E<B_{2} / h>
$$

Equations (12) and (17) are straightforward flux surface averages of the continuity equation (1) and the $\vec{B}$ component of Ohm's Law (4), respectively. Equations (13) and (14) are averages of the equations obtained upon dotting the momentum equation (2a) with $\vec{B} / n M$ and $R \hat{z}$, respectively. The former averaging is carried out in Appendix A. To obtain the latter, use is made of the fact that $f \vec{j} \cdot d \vec{s}=0$, which is a consistency condition on equation (5a), anci of the geometrical identity $\overrightarrow{A A}: \vec{\nabla} \hat{z h} \equiv 0$. Equations (15) and (16) are obtained by annihilating the appropriate leading order term in the heat equations (3); this is done in Appendix B. Equations (12)-(17) 
are evaluated to lowest order in $\left(v_{\mathrm{p}} / \Theta C_{s}\right)$ for simplicity. (The symbol $\simeq$ i:ldicates which equations depend on this expansion). Equations (12) and (14) express the conservation of mass and toroidal angular momentum. Equation ( 13$)$ is a statement of the rate of change of circulation of flow on a magnetic surface. The circulation is defined as the parallel. flow integrated over an entire field line, namely $\oint d \vec{l} \cdot \vec{v}$ where $d \vec{l}$ is along a field line. It is readily shown that the quantity $\langle\vec{v} \cdot \vec{B}\rangle$ appearing in (13) is proportional to the circulation viz. $\left(\int \mathrm{d} S /|\vec{\nabla} \mathrm{p}|\right)^{-1} \int(\mathrm{d} S /|\vec{\nabla} \mathrm{p}|) \overrightarrow{\mathrm{v}} \cdot \overrightarrow{\mathrm{B}} \equiv(\oint \mathrm{d} \ell / \mathrm{B})^{-1} \oint(\mathrm{d} \ell / \mathrm{B}) \overrightarrow{\mathrm{v}} \cdot \overrightarrow{\mathrm{B}}$. (This definition applies only for closed lines. For ergodic lines, appropriate limits are to be taken). Equation (13) expresses the fact that the circulation per magnetic surface, in addition to being convected from surface to surface, is also aestroyed by the dissipative mechanisms of resistivity, viscosity and electron and ion thermal conductivities (expressed term by term in that order on the right hand side; note that the contribution of the last term is related to a departure of the species from "perfect" adiabaticity or isothermality). Likewise, the species' entropy (Eqs. (15) and (16)) is changed by viscous and conduction effects in addition to convective flows, Equation (17) relates the parallel current to the external electric field.

All terms in Eqs. (12)-(17) involving any of the dissipation coefficients $i, n_{0}, k$ or a time derivative a/at may be explicitly evaluated using the zero order equilibrium quantities (6)-(11) (since our expansion requires $\left.\partial / \partial t \sim\left(n, n_{0}, k\right) \sim O(\ell / q R)\right)$. The only remaining quantities 
to be evaluated are those involving the radial flux of some $f$, $\int \mathrm{d} \overrightarrow{\mathrm{S}} \cdot \vec{v} f$, and the quantity $\langle\mathrm{TB} \cdot \vec{\nabla} \mathrm{s}\rangle$ for each species. For the former, from the identity $\left(\int \mathrm{d} \overrightarrow{\mathrm{S}} /|\vec{\nabla} \mathrm{p}|\right)^{-1} \int \mathrm{d} \overrightarrow{\mathrm{S}} \cdot \overrightarrow{\mathrm{v} f} \equiv \mathrm{p}^{\prime}(r)\left\langle f \mathrm{v}_{r} / \mathrm{h}_{\mathrm{r}}\right\rangle$, the quantity $\left\langle f v_{r} / h_{r}\right\rangle$ is required. This quantity is obtained by suitably averaging the $\hat{z}$ component of Ohm's Law (4) and using Eq. (17). This procedure is outlined in Appendix C. The result is

$$
\left\langle f v_{r} / h_{r}\right\rangle \simeq v_{D}\left[D\langle f\rangle+\theta^{-2}\left(\left\langle f h^{2}\right\rangle-\langle f\rangle\left\langle h^{-2}\right\rangle^{-1}\right)\right]
$$

where $v_{D}, D$ and $\langle f\rangle$ are defined further in equations (26) and (27). For the other required quantity, $\langle\overrightarrow{T B} \cdot \vec{\nabla} \mathrm{s}\rangle$, the deviation of $s$ from perfect adiabaticity for of $T$ from perfect isothermality, as the case may be) is needed. This is obtained by solving for $s$ to first order in $k$ using the heat. equation (3) (or solving for $T$ to first order in $1 / K$ ). These computations are outlined in Appendix D. In this part of the calculation and in the term involving $\kappa$ in (15), inertial corrections to the equilibrium pressure are needed; these, too, are calculated in Appendix D. The results for electrons are: (1) Adiabatic electrons

$$
\left\langle\left(T_{e} / M\right) \vec{B} \cdot \vec{\nabla} s_{e}\right\rangle \simeq-B_{o}\left(v_{p} / \theta\right)\left(\theta C_{s} / v_{p}\right)^{2}\left(\kappa_{e} / n\right)\left\langle\left(\vec{B} \cdot \vec{\nabla}_{e_{e}} / B T_{e}\right)^{2}\right\rangle
$$

where

$$
\begin{gathered}
(5 / 2)\left(1+T_{i} / T_{e}\right)\left(T_{e} / M\right)=\text { const. }-\left(v_{p}^{2} / h^{2} b_{z}^{2}-v_{E}^{2} h^{2}\right) / 2 \theta^{2} \\
c_{s}^{2} \equiv\left(T_{e} / M\right) .
\end{gathered}
$$


(2) Isothermal electrons, adiabatic ions

$$
\left\langle\left(T_{e} / M\right) \vec{B} \cdot \vec{\nabla}_{s} s^{>}=-B_{0}^{-1}\left(v_{p} / \theta\right)\left(n C_{s}^{2} / k_{e}\right)<B^{2}\left(\ln n-\left\langle B^{2} \ln n\right\rangle /\left\langle B^{2}\right\rangle\right)^{2}\right\rangle
$$

where

$$
\left[1+(5 / 3)\left(T_{i} / T_{e}\right)\right]\left(T_{e} / M\right) \ln n \simeq \text { const. }-\left(v_{p}^{2} / h^{2} b_{z}{ }^{2}-v_{E}{ }^{2} h^{2}\right) / 2 \theta^{2}
$$

The ion contribution $\left\langle\mathrm{T}_{i} \vec{B} \cdot \vec{\nabla} s_{i}\right\rangle$ may be evaluated similarly. It is, however, easy to verify that this quantity is always smaller than the viscous term in equation (13) by a factor $\left(v_{\mathrm{p}} / \theta \mathrm{c}_{\mathrm{s}}\right)^{2}$. Thus, we may neglect the ion contribution arising from thermal conduction.

Equations $(12)-(16)$ and $(18)-(20)$ supplemented by equations (6)-(11), form a complete set of equations from which the time evolution of the flow profiles $v_{E}(r)$ and $v_{p}(r)$ may be determined. As mentioned before, this set is to be understood to be complete only insofar as $\vec{E}$ may be taken to be curl free and perpendicular electron and ion heat transport may be neglected. In the next section, Eqs. (6)-(11) are substituted into $(12)-(20)$ and the resulting set is simplified by an inverse aspect ratio expansion. 
VII. INVERSE ASPECT RATIO EXPANSION

The equilibrium relations (6)-(11) are substituted into Eqs. (12)-(16) and (18)-(20) and all surface averages are carried out to lowest significant order in the inverse aspect ratio $\varepsilon \equiv\left\langle h_{\theta}\right\rangle / R$. Specifically, we assume $h=1+O(\varepsilon)$ in carrying out the averages. The resulting equations include surface averages of quantities that are only dependent on the shape of the minor cross section. These various averages are redefined to be proporticnal to coefficients $\alpha_{i}$ in such a way that each $\alpha_{i}$ is equal to uiity for circular cross sections (for which $r$ and $\theta$ become polar coordinates to lowest order in $\varepsilon)$. If the non-circularity is not too extreme the $\alpha_{i}$ 's may be expected to be of order unity for non-circular cross sectional shapes. The final equations are:

$$
\partial n / \partial t+\nabla_{L^{n}} n v_{D}\left(D+2 q{ }^{2} \alpha_{1}\right)=0
$$


$-18-$

$(\partial / \partial t)\left[v_{T}+\left(\alpha_{3}+2 q^{2} \alpha_{1}\right) \theta v_{p}\right]$

$$
\begin{aligned}
& +\nabla_{r} v_{D}\left\{\left(D+2 q^{2} \alpha_{1}\right)\left[v_{T}+\left(\alpha_{3}+2 q^{2} \alpha_{1}\right) \theta v_{p}\right]-2 q{ }^{2} \alpha_{1}\left(v_{p} / \theta\right)\right\} \\
& =A_{r_{i}}-(3 / 2) \alpha_{4}\left(n_{0} / n M R^{2}\right) \theta v_{p}
\end{aligned}
$$

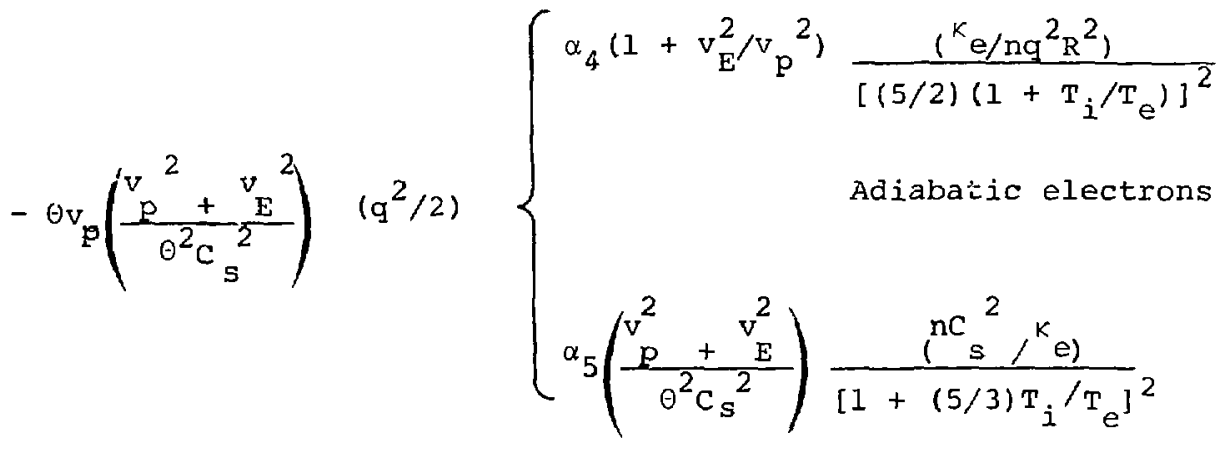

(22)

$(\partial / \partial t)\left(n v_{T}\right)$

$$
\begin{gathered}
+\nabla_{r_{D}}\left[\left(D+2 v^{2} \alpha_{I}\right) v_{T}+2 q^{2} \alpha_{2}\left(v_{T}-v_{p} / \theta\right)\right]=0 \\
\cdot \quad \partial s_{i} / \partial t+s_{i} \cdot v_{D}\left(D+2 q^{2} \alpha_{1}\right) \\
=(3 / 2) \alpha_{4}\left(n_{0} / n M R^{2}\right)\left(v_{p} / C_{s}\right)^{2}
\end{gathered}
$$




$$
\begin{aligned}
& \partial s_{e} / \partial t+s_{e} v_{D}\left(D+2 q^{2} \alpha_{1}\right)
\end{aligned}
$$

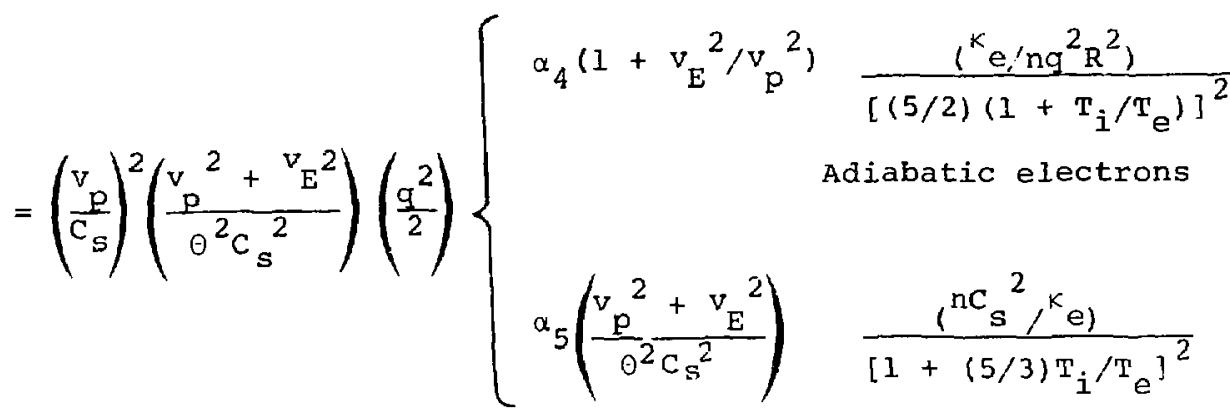

$$
\begin{aligned}
& \text { Isothermal electrons }
\end{aligned}
$$

where

$$
\begin{aligned}
& v_{D} \equiv-n p^{\prime} / E_{O} \\
& \left.D \equiv-\left(B_{O_{0}} B_{0}^{\prime} / p^{\prime}\right)<1 / h^{2} h_{r}^{2}>/<1 / h^{2}\right\rangle \\
& q \equiv\left\langle h_{\theta}>/ \theta R \equiv \varepsilon / \theta\right. \\
& v_{T} \equiv\left\langle h_{z}\right\rangle \equiv \theta^{-1}\left(v_{p}-v_{E}<h^{2}>\right)
\end{aligned}
$$

The quantity $\mathrm{v}_{\mathrm{T}}$ is a surface function proportional to the toroialal angular momentum and defined in terms of $v_{p}$ and $v_{E}$. $v_{D}$ is the resistive "classical flow" and $q$ is the "safety factor:" The averaging operator $\langle f\rangle$ and the radial divergence operator $\nabla_{r}$ 
are defined as

$$
\begin{aligned}
& \langle f\rangle \equiv(\oint \mathrm{d} \theta J)^{-1} \oint \mathrm{d} \theta \cdot J f \\
& \nabla_{\mathrm{r}} f \equiv(\oint d \theta J)^{-1}(\partial / \partial x) \oint d \theta J f
\end{aligned}
$$

The quantity $A_{n}$ includes resistive contributions and is given by

$$
\begin{aligned}
A_{\eta} & \equiv-v_{T}\left\langle\left(n B_{0} \cdot h_{\theta}\right) \cdot / h_{\theta} B_{0}\right\rangle \\
& +v_{p}<\left[n h_{\theta}^{-1}\left(\theta h_{\theta} B_{0}\right)^{\prime}\right] \cdot / B_{O} \\
& +\left(1-h^{2} /<h^{2}>\right)\left(n h_{\theta} B_{0}\right)^{\prime} / \theta h_{\theta} B_{0}>
\end{aligned}
$$

Finally the $\alpha_{i}$ 's are defined as follows:

$$
\begin{aligned}
2 \varepsilon^{2} \alpha_{1} & \equiv\left\langle h^{2}\right\rangle-\left\langle 1 / h^{2}\right\rangle-1 \\
2 \varepsilon^{2} \alpha_{2} & \equiv\left\langle h^{4}\right\rangle\left\langle h^{2}\right\rangle^{-1}-\left\langle h^{2}\right\rangle \\
\alpha_{3} & \equiv\left\langle 1 / h^{2} h_{r}{ }^{2}\right\rangle \\
\alpha_{4} / 2 R^{2} & \equiv\left\langle J^{-2}\left(\partial \ell n h b_{z} / \partial \theta\right)^{2}\right\rangle \\
\varepsilon^{2} \alpha_{5} & \equiv 2\left\langle(h-1)^{2}\right\rangle
\end{aligned}
$$


The set (21)-(29) now completely describes the time evolution of the equilibrium for time scales faster than confinement times. On the right hand sides of the circulation and entropy equations, the subdominant terms have been suppressed for clarity. These include, for example, contributions due to electron viscosity or ion thermal conductivity.

We note the following features of the foregoing equations. First, we note that the convection term in (21) includes botin the "classical flux" and the $\mathrm{q}^{2}$ enhancement due to the "PfirschSchlüter" flow (D 1 for $\beta_{\theta}^{\sim 1}$ ). Iikewise, (22) and (23) respectively show that the circulation $v_{T}+\left(1+2 q^{2} \alpha_{1}\right) \theta v_{p}$ and the angular momentum $\mathrm{nMRv}_{\mathrm{T}}$ are also directly convected by these two flows; however, there is an additional contribution to the rate of change of these latter two quantities which results from a coupling between the Pfirsch-Schliter flow and $v_{p}$ - This additional contribution is larger than the direct convection by a factor of $\theta^{-1}$. This coupling is responsible for the stringer spin-up. The second fact of interest is that the circulation is destroyed by viscous effects only if $\mathrm{v}_{\mathrm{p}} \neq 0(\mathrm{Eq} \cdot(22))$. Thus a poloidal component to the flow pattern is crucial for the viscous dissipation associated with magnetic pumping. Finally, we note the correspondence in the terms on the right hand sides of the circulation equation (22) and the entropy equations (24) and (25). This simply reflects conservation of energy: as the circulation damps, the plasma heats up correspondingly. 
VIII. TIME EVOLUTION OF FLOW

As set forth in the introduction, we are mainly interested in time scales shorter than $(\mathrm{m} / \mathrm{M})^{1 / 2}{ }_{\mathrm{T}} \mathrm{ps}$ where

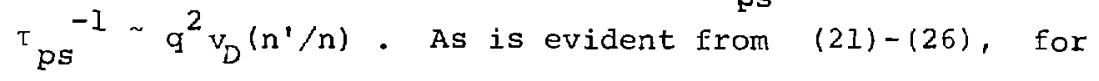
these short times we may ignore the time evolution of the density, and that evolution of the circulation, angular momentum, and entropy produced by direct classical and Pfirsch-Schlitter convection. Furthermore, the effect of the resistive terms $A_{\eta}$ may also be estimated to be small during these times. For simplicity, we restrict ourselves to circular cross sectiors $\left(\alpha_{i}+1\right)$. Upon substituting for $\partial v_{T} / \partial t$ in (22) from (23) and making the stated approximations, we obtain the time evolution of $v_{\mathrm{p}}$ and $v_{T}$ from (22) and (23) viz.

$$
\begin{aligned}
& \left(1+1 / 2 q^{2}\right) \partial \ln v_{p} / \partial t \\
& \approx-\theta^{-2} v_{D}\left(n^{\prime} / n\right)-(3 / 4)\left(n_{0} / n M q^{2} R^{2}\right)
\end{aligned}
$$

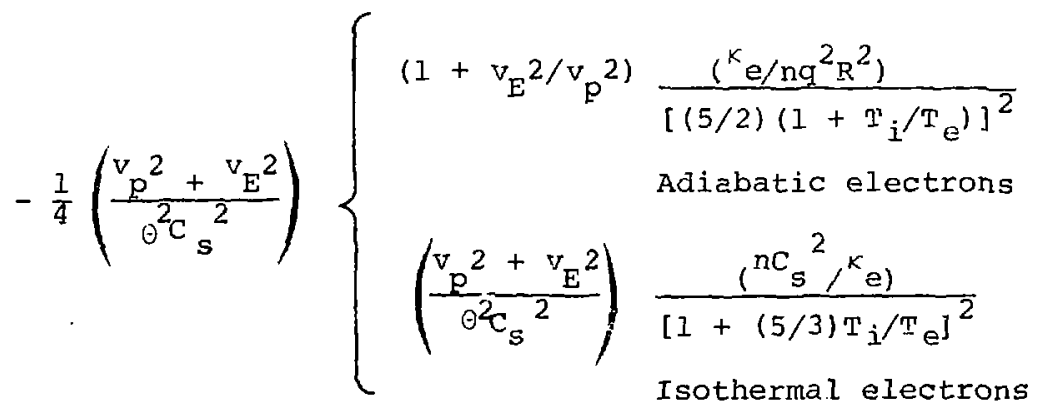




$$
\partial v_{T} / \partial t \approx 2(n r)^{-1}(\partial / \partial r)\left(n r q^{2} v_{D} v_{p} / \theta\right)
$$

We may assume $\partial \mathrm{n} / \partial t \approx 0$ and, for the moment, we ignore any changes in temperature i.e., $\partial \mathrm{T} / \partial \mathrm{t} \approx 0$. Equation (30) yields the rate of change of poloidal rotation. The terms on the right hand side represent, respectively, the destabilization due to resistivity in a time $\theta^{2} \mathrm{q}^{2} \tau \mathrm{p}_{\mathrm{s}}$ ) and the stabilization due to viscosity (magnetic pumping) and thermal conduction. These effects add independently to alter the flows. From $^{13}$ $n_{0}=.96 \mathrm{nT} / v_{i i}$, the damping rate due to magnetic pumping may be estimated as

$$
\gamma \simeq(3 / 4)\left(1+1 / 2 q^{2}\right)^{-1}(\ell / q R)^{2} v_{i i}
$$

From $^{13} \mathrm{k}_{\mathrm{e}}=3 \cdot 2 n \ell v_{i j}(\mathrm{~m} / 2 \mathrm{M})^{-1 / 2}$, the damping rate due to thermal conduction may also be estimated. For adiabatic electrons, or $\ell / q R<(m / M)^{1 / 2} v_{p} / \theta C_{s}$, the rate is

$$
\gamma \simeq(3 \cdot 2 / 25)\left(1+1 / 2 q^{2}\right)^{-1} v_{i i}(l / q R)^{2}\left(v_{p} / \theta C_{s}\right)^{2}(m / 2 M)^{-1 / 2}
$$

while for isothermal electrons, i.e., (m/M) $1 / 2 v_{p} / \theta C_{s}<\ell / q R$, 
the rate is

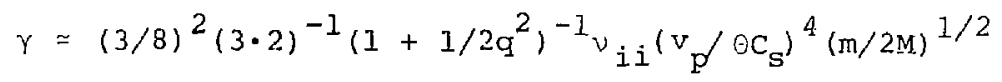

(In obtaining the foregoing damping rates, we have assumed that $\mathrm{T}_{\mathrm{e}}=\mathrm{T}_{i}$ and $\mathrm{v}_{\mathrm{E}} \sim \mathrm{v}_{\mathrm{p}^{*}}$ Each of these damping mechanisms is dominant under different conditions. This yields the parameter space plot of Fig. 2 which schematically shows the conditions under which thest various effects are dominant. (We have used $\left.\tau_{p s}{ }^{-1} \simeq q^{2} \rho^{2} v_{i i}(m / M)^{1 / 2} / a.\right)$ Having obtained these rates of change, it may be verified for each region separately that the temperature profiles change at a rate slower than the poloidal damping rate by a ractor of $2 q^{2}\left(v_{p} / c_{s}\right)^{2}$. This reflects energy conservation: the energy per particle associated with poloidal rotation is approxirately $M q^{2} v_{p}^{2}$ (see section $I X$ ) and so $\partial T / \partial t \simeq-(\partial / \partial t)\left(M q^{2} v_{p}^{2}\right.$ ). Thus temperature time variations may be neglected in the above equations.

Equation (31) shows that the time scale for the rate of change of the toroidal angular momentum per surface is ${ }^{\Theta \tau}$ ps 
IX. HEURISTIC DERIVATION

The damping rates given in $\mathrm{Eq} \cdot$ (30) while correct are the result of a fairly involved mathematical deduction. It is desirable to arrive at them qualitatively by a more simple physical argument. In this section we give a heuristic derivation of the damping due to magnetir pumping. Magnetic pumping is effected as the plasma rotates through the inhomogeneous toroidal magnetic field. This heats up the plasma and leads to an irreversible transformation of the kinetic ene , Uf rolation into thormal e I wich damps the rotation. The heating of the plasma may be visualized as follows.

Consider an element of fluid rotating about the minur cross section of the plasma (Fig. 4). Because of flux preservation, its cross section transverse to the magnetic field is smaller on the inside of the torus than on the outside by a relative amount of order $\varepsilon=a / R$. At the same time, because it ' 3 incompressible, its extension along the magnetic flux tube must vary in the opposite sense (see Figure). Thus, as an element moves from the outside of the torus to the inside, it constricts in a transverse dimension and distends in the dimension parallel to the flux tube. Accompanying the transverse constriction is a tendency for the perpendicular temperature $T_{1}$ to increase by a factor of order $\varepsilon^{-1}$ las a result of the conservation of the adiabatic invariant $\mu \nu$, and accompanying the distention is a tendency for the parallel temperature $T_{\|}$ 
to decrease by a factor of order $\varepsilon$ (fxom conservation of the second adiabatic invariant J). But the collision frequency $v$ is much greater than the rotation rate $v_{p} / a$ so that at all times, $T_{1}$ and $T_{11}$ are kept nearly equal by a continious transfer of energy from $T_{\perp}$ to $T_{11}$. In fact, for $\nu \rightarrow \infty$, $T_{\perp}$ and $T_{\|}$are exactiy equal and, since the fluia element as a whole is incompressible, no net work is imparted to the element. However, since collisions are not infinitely frequent, the energy transfer from $T_{1}$ to $T_{\|}$lags the infinite collision transfer rate by a factor of $\mathrm{v}_{\mathrm{p}} / \mathrm{av}$. As a result of this lag, $T_{\perp}$ is maintained at a slightly hotter level than tire mean, and $T_{\|}$at a slightly cooler level. In fact the maximum relative difference ketween $\mathrm{T}_{\perp}$ and $\mathrm{T}_{\|}$is of order $\left(v_{p} / a \nu\right) \varepsilon$, where $\varepsilon$ represents the fractional cnange in energy in the absence of collisions. Now, in an exactly analogous way, the opposite circumstance prevails in the second half of the rotation cycle when the element moves from the inside to the outside. Namely, $\mathrm{T}_{\perp}$ is maintained slightly cooler and $T_{\|}$slightly hotter. As a result of these lags, the deviation of $T_{\perp}$ from "ideal" is directly in phase wj.th the perpendicular pumping of the flux tube and, in effect, the perpendicular contraction does more work on the plasma in the first half of the cycle than it extracts from it in the second half. The net energy deposited into tine plasma per particle per cycle is then of order $\varepsilon^{2}\left(v_{p} / a v\right) T$. In a 
directiy analogous manner, the parallel contraction and distention acts to deposit energy into the plasma at a rate comparable to the foregoing rate. Thus the net work done per particle per unit time is roughly

$$
\mathrm{dw} / \mathrm{d} t \sim \varepsilon^{2}\left(v_{\mathrm{p}} / \mathrm{a}\right)^{2} v^{-1} \mathbf{T}
$$

This work must. come from the rotational energy of the flux tube. The rotational energy is made up of the mean rotation $v_{p}$ together with the energy associated with the accompanying parallel distentions which take place at a speed of order $q v_{p}$. (This may be seen from $\vec{\nabla} \cdot \vec{v}_{\|}=-\vec{\nabla} \cdot \vec{v}_{\perp}$ ). Equating the rotational energy loss with the temperature rise

$$
(j / \partial t)(1 / 2) M v_{p}^{2}\left(1+2 q^{2}\right)--\varepsilon^{2}\left(v_{p} / a\right)^{2} v^{-1} T
$$

gives, upto numerical factors, the poloidal damping rate of Eq. (32). (The factor of 2 in the $q^{2}$ term may be derived from more detailed considerations).

Thus, we may identify the damping of poloidal rotation by viscosity with the phenomenon of heating the ions by magnetic pumping. (The electron heating is small since $v_{e}>v_{i}$ ). We add parenthetically that the damping of rotation which arises from parallel thermal conduction may be identified as a more generalized magnetic pumping phenomenon. 


\section{CONCLUSION}

We have consiciered subsonic flows about an axisymmetric magnetostatic tokamak equilibrium with arbitrary minor cxoss section. Differential equations for the slow time evolution of these poloidal and toroidal flows have been derived in the collisional limit (Eqs. (12)-(20) ). We have specialized these equations in the limit of small inverse aspect ratio [Eqs. (21)-(29)] and, further, to circular cross sections [Eqs. (30)-(31) ]. The time rates of change of the poloidal Elow have been estimated for various parameter regimes (Fig. 2). A general conclusion to be drawn from our investigation is that for most cases of practical interest, the process of magnetic pumping rapidly damps any poloidal flow, at least to a level comparable with the diamagnetic speed. ACKNOWLEDGEMEITS

We are happy to acknowledge helpful discussions with A. H. Boozer, C. Oberman, T. H. Stix and A. A. Ware. This work was supported by the United States Department of Energy Contract no. EY-76-C-02-3037. 
APPENDIX A. DERIVATION OF THE RATE OF CHANGE OF CIRCULATION

We derive equation (13). Dotting (2a) with $\vec{B} / n M$ and averaging yields

$$
(\partial / \partial t)\langle\vec{B} \cdot \vec{v}\rangle=-\left\langle(n M)^{-1} \vec{B} \cdot(\vec{\nabla} p+\vec{\nabla} \cdot \vec{M}+n M \vec{V} \cdot \vec{\nabla} \vec{v})\right\rangle
$$

Evaluating term by term on the right hand side,

$$
\begin{aligned}
& \left\langle\mathrm{n}^{-1} \overrightarrow{\mathrm{B}} \cdot \vec{\nabla} \mathrm{p}\right\rangle=\sum_{i, \mathrm{e}}\langle\mathrm{T} \overrightarrow{\mathrm{B}} \cdot \vec{\nabla} \ln \mathrm{n}\rangle \\
& =-\sum_{i, e}\langle\mathrm{~T} \vec{B} \cdot \vec{\nabla} \mathrm{s}\rangle \\
& \left\langle(n M)^{-1} \vec{B} \vec{\nabla}: \overleftrightarrow{\Pi}\right\rangle \simeq(n M)^{-1}\langle\stackrel{t}{\langle\Pi}: \vec{\nabla} \vec{B}\rangle \\
& =-\left(3 \eta_{0} / n M\right)\left\langle B^{-2} \vec{B} \vec{B}: \vec{\nabla} \vec{V} \vec{B} \cdot \vec{\nabla} \ell n B\right\rangle \\
& \simeq-\left(3 \eta_{0} / n M\right)\langle\vec{v} \cdot \vec{\nabla} \ell n B \quad \vec{B} \cdot \vec{\nabla} i \cdot 2 B\rangle
\end{aligned}
$$

where the last step follows by noting that $\vec{v}$ may be written in the form $\vec{v} \simeq f(p) \vec{B}+(d \phi / d p) \vec{j}$. Only the lowest order terms in $\left(v_{p} / \theta C_{s}\right)$ have been retained. 


$$
\begin{aligned}
\langle\overrightarrow{\mathrm{B}} \overrightarrow{\mathrm{v}}: \vec{\nabla} \overrightarrow{\mathrm{v}}\rangle & =\left\langle\overrightarrow{\mathrm{B}} \cdot\left[\vec{\nabla} \mathrm{v}^{2} / 2-\overrightarrow{\mathrm{v}} \times(\vec{\nabla} \times \overrightarrow{\mathrm{v}})\right]\right\rangle \\
& =\langle\vec{\nabla} \cdot(\overrightarrow{\mathrm{B}} \times \overrightarrow{\mathrm{v}}) \times \overrightarrow{\mathrm{v}}+\overrightarrow{\mathrm{v}} \cdot \vec{\nabla} \times(\overrightarrow{\mathrm{v}} \times \overrightarrow{\mathrm{B}})\rangle \\
& =\left(\int \mathrm{dS} /|\vec{\nabla} \mathrm{p}|\right)^{-1}(\partial / \partial \mathrm{p}) \int \mathrm{d} \overrightarrow{\mathrm{S}} \cdot \overrightarrow{\mathrm{v} v} \overrightarrow{\mathrm{B}} \\
& +\langle\overrightarrow{\mathrm{v}} \cdot \vec{\nabla} \times(\eta \vec{j}-\hat{\mathrm{V}} \mathrm{E} / \mathrm{h})\rangle
\end{aligned}
$$

Substitution of (A2) - (A4) into (AI) yields the desired result Eq. (13) .

APPENDIX B. HEAT EQUATIONS

We sketch the derivation of Eqs. (15) and (16) starting from Eq. (3). The adiabatic case, Eq. (15), is treated in part (a) below and the isothermal case in (b).

(a) Adiabatic Case:

The leading order term in $(3 a)$ is $\vec{v} \cdot \vec{\nabla} s$ giving $s=s(r)$ to lowest order. We annihilate this term by dividing (3a) by $T$ and averaging. This yields

$$
\begin{aligned}
& \langle\mathrm{n}\rangle \partial \mathrm{s} / \partial t+\langle\mathrm{nv} \cdot \vec{\nabla} \mathrm{s}\rangle \\
& =-\langle(\vec{\nabla} \cdot \overrightarrow{\mathrm{q}}+\overleftrightarrow{\Pi}: \vec{\nabla} \vec{v}) / \mathrm{T}\rangle
\end{aligned}
$$


Evaluating term by term,

$$
\begin{aligned}
\langle\mathrm{n} \overrightarrow{\mathrm{v}} \cdot \vec{\nabla} \mathrm{s}\rangle & =\langle\mathrm{n} \overrightarrow{\mathrm{v}} \cdot \vec{\nabla} \mathrm{p}\rangle(\partial \mathrm{s} / \partial \mathrm{p}) \\
& =\left(\int \mathrm{ds} /|\vec{\nabla} \mathrm{p}|\right)^{-1}(\partial \mathrm{s} / \partial \mathrm{p}) \int \mathrm{d} \overrightarrow{\mathrm{s}} \cdot \mathrm{n} \overrightarrow{\mathrm{v}} \\
\langle\vec{\nabla} \cdot \overrightarrow{\mathrm{q}} / \mathrm{T}\rangle= & -\left\langle\mathrm{T}^{-1} \overrightarrow{\mathrm{B}} \cdot \vec{\nabla}\left(k \mathrm{~B}^{-2} \overrightarrow{\mathrm{B}} \cdot \vec{\nabla} \mathrm{T}\right)\right\rangle \\
= & -k\left\langle(\overrightarrow{\mathrm{B}} \cdot \vec{\nabla} \mathrm{T} / \mathrm{BT})^{2}\right\rangle \\
\langle\overleftrightarrow{\mathrm{I}}: \vec{\nabla} \overrightarrow{\mathrm{v}} / \mathrm{T}\rangle=- & \left\langle\left(3 \eta_{0} / \mathrm{T}\right\rangle[(\hat{\mathrm{bb}}-\stackrel{4}{1} / 3): \vec{\nabla} \overrightarrow{\mathrm{v}}]^{2}\right\rangle \\
\simeq & \left\langle\left(3 \eta_{0} / \mathrm{T}\right\rangle(\overrightarrow{\mathrm{v}} \cdot \vec{\nabla} \ell \mathrm{nB})^{2}\right\rangle
\end{aligned}
$$

Substitution of (B2) - (B5) into (B1) yields (15).

(b) Isothermal Case:

Here we formally expand in the small parameter (rutation rate/relaxation rate) $\sim 1 / k$. The conduction term in (3a), $\vec{\nabla} \cdot \vec{q}$, is then considered to be zero order, the convective term $\vec{v} \cdot \vec{\nabla} s$ first order, and the remaining terms are (maximally) brought in at second order. The lowest order solution is then $T=T(r)$. It is next readily observed that the consistency condition to solve for $\mathrm{T}^{(1)}$ is trivially satisfied. Finally, the condition to solve for $\mathrm{T}^{(2)}$ is what yields Eq. (16). This is obtained upon averaging (3a) which yields

$$
\mathrm{T}\langle\mathrm{n} \partial \mathrm{s} / \partial \mathrm{t}\rangle+\langle\mathrm{nT} \overrightarrow{\mathrm{v}} \cdot \vec{\nabla} \mathrm{s}\rangle(1)=-\langle\vec{\Pi}: \vec{v} \vec{v}\rangle
$$


where the superscript (1) denotes the term which is to be evaluated to first order. Now

$$
\begin{aligned}
\langle n \mathrm{nT} \cdot \vec{v} \mathrm{~s}\rangle(1) & =\langle\mathrm{nT}(1) \overrightarrow{\mathrm{v}} \cdot \vec{\nabla} \mathrm{s}\rangle+\mathrm{T}\langle(\mathrm{n} \overrightarrow{\mathrm{v}})(1) \vec{\nabla} \mathrm{s}\rangle \\
& \simeq\left(n v_{\theta} / \mathrm{B}_{\theta}\right)\langle\mathrm{T}(1) \overrightarrow{\mathrm{B}} \cdot \vec{\nabla} \mathrm{s}\rangle \\
& +\mathrm{T}\langle\mathrm{n} \overrightarrow{\mathrm{v}} \cdot \vec{\nabla} \mathrm{p}\rangle\langle\partial \mathrm{s} / \partial \mathrm{p}\rangle
\end{aligned}
$$

where we have used the fact that $(\partial / \partial \theta)\left(n_{v_{\theta}} / B_{\theta}\right)=0$ to lowest order, and expanded the second term to lowest order in $\left(v_{p} / \Theta C_{s}\right)^{2}$. Evaluating the term $\langle\overrightarrow{\|}: \vec{\nabla} \vec{v}\rangle$ as in (B5) and using (B7) in (B6) gives the desired result (16).

\section{APPENDIX C. THE RADIAL FLUX}

We wish to solve for the quantity $\left\langle f_{v_{r}} / h_{r}\right\rangle$ in terms of the lowest order equilibrium quantities.

$$
\text { Multiplying the } \hat{z} \text { component of ohm's Law (4) by }\left(f / h_{r} B_{\theta}\right)
$$
and averaging yields

$$
\left\langle f v_{r} / h_{r}\right\rangle=\left\langle\left(n j_{z}-\varepsilon / h\right)\left(f / h_{r} B_{\theta}\right)\right\rangle
$$

We now make use of equations (17), (6) and (9) to substitute for the quantities $\varepsilon^{\circ}, B_{\theta}, B_{z}, j_{z}$ and $j_{\|}$. This gives 


$$
\begin{aligned}
\left\langle f v_{r} / h_{r}\right\rangle \simeq & \left(n / \theta^{2} B_{o}\right)\left[-\left\langle\left(B_{o}^{\prime}+p^{\prime} h^{2} / B_{o}\right) f>\right.\right. \\
& +\left\langle I / h^{2}>-1<B_{o} / / h^{2} b_{z}^{2}-p^{\prime} / B_{o}\right\rangle\langle f>]
\end{aligned}
$$

Using $b_{z}^{-2}=1+\theta^{2} / h_{r}^{2}$ and rearranging gives the desired result

$$
\left\langle f v_{r} / h_{r}>\simeq v_{D}[D<f\rangle+\theta^{-2}\left(\left\langle f h^{2}>-\langle f\rangle\left\langle 1 / h^{2}\right\rangle^{-1}\right)\right]\right.
$$

where $D$ and $v_{D}$ are defined in the text.

APPENDIX D. DERIVATION OF EQUATIONS (19) AND (20)

We evaluate the quantity $\langle\overrightarrow{T B} \cdot \vec{\nabla} s\rangle$. Note that this is zero when the species is either perfectly adiabatic, $s=s(r)$, or perfectly isothermal, $T=T(r)$. Thus contributions arise from any departures from perfect adiabaticity or isothermality. Consider the adiabatic case first.

(a) Adiabatic Case:

Denoting the departure by $\tilde{s}$, the equation determining $\tilde{\mathbf{s}}$ is given by the heat equation (3a) viz

$$
\begin{aligned}
\overrightarrow{\mathrm{v}}_{\theta} \cdot \vec{\nabla}_{s}{ }^{2} & -(\mathrm{nT})^{-1} \vec{\nabla} \cdot \overrightarrow{\mathrm{q}}-(\mathrm{nT})^{-\mathrm{It}_{\mathrm{I}} t: \vec{\nabla} \vec{v}} \\
& -\left(2 s / \partial t+s^{\prime} v_{r} / h_{r}\right)
\end{aligned}
$$


where all terms on the right hand side are evaluated using the equilibrium quantities (6)-(11). Noting that $(\partial / \partial \theta)\left(B_{\theta} / n v_{\theta}\right)=0$ for the equilibrium, we multiply both sides by $\mathrm{TB}_{\theta} / \mathrm{v}_{\theta}$ and average to obtain the desired quantity

$$
\langle\mathrm{TB} \cdot \vec{\nabla} \tilde{S}\rangle=-\left(\mathrm{B}_{\theta} / \mathrm{n} v_{\theta}\right)\left\langle\vec{\Pi}: \vec{\nabla} \vec{v}+\mathrm{n} \mathrm{\eta}\left(\partial \mathrm{s} / \partial \mathrm{t}+\mathrm{s}^{\prime} \mathrm{v}_{\mathrm{r}} / \mathrm{h}_{\mathrm{r}}\right)\right\rangle
$$

Using the consistency condition (15) and evaluating to lowest order in $\left(\mathrm{v}_{\mathrm{p}} / \theta \mathrm{C}_{\mathrm{s}}\right)$, the right hand side may be simplified to give

$$
\langle\overrightarrow{\mathrm{T}} \cdot \vec{\nabla} \mathrm{S}\rangle=-\left(\mathrm{B}_{\beta} / \mathrm{nv}_{\theta}\right) \mathrm{T}\left\langle\mathrm{K}(\overrightarrow{\mathrm{B}} \cdot \vec{\nabla} \mathrm{T} / \mathrm{BT})^{2}\right\rangle
$$

The equilibrium equations may be utilized once more to yield (19a).

\section{Isothermal Case:}

Here the temperature is driven away from isothermality by the poloidal rotation. This is given by equation (3a) which, to first oxder in $1 / k$, may be written

$$
-\vec{\nabla} \cdot \vec{q} \equiv \vec{B} \cdot \vec{\nabla} \times B-2 \vec{B} \cdot \vec{\nabla} \tilde{r}^{2}=n T \vec{v}_{\theta} \cdot \vec{\nabla} s
$$

where $\tilde{T}$ represents the departure from isothermality, Once again, utilizing $(\partial / \partial \theta)\left(B_{\theta} / n v_{\theta}\right)=0$, we may integrate once to obtain

$$
\kappa B^{-2} \vec{B} \cdot \vec{\nabla} \tilde{T}=\left(n v_{\theta} / B_{\theta}\right) T\left(s-\left\langle B^{2} s\right\rangle /\left\langle B^{2}\right\rangle\right)
$$


where the constant of integration is determined by demanding $\langle\overrightarrow{\mathrm{B}} \cdot \vec{\nabla} \tilde{\mathrm{T}}\rangle=0$. The desired quantity $\langle\overrightarrow{\mathrm{TB}} \cdot \vec{\nabla} \mathrm{s}\rangle$ may be rewritten, using $(3 b)$, as

$$
\langle\mathrm{TB} \cdot \vec{\nabla} s\rangle=\langle\ln n \overrightarrow{\mathrm{B}} \cdot \vec{\nabla} \mathrm{T}\rangle
$$

whereupon, using (D5), we obtain

$$
\begin{aligned}
& \langle\ln n \vec{B} \cdot \vec{\nabla} \mathrm{T}\rangle=k^{-1} \mathrm{~T}\left(n v_{\theta} / \mathrm{B}_{\theta}\right)\left\langle\mathrm{B}^{2} \operatorname{lnn}\left(s-\left\langle\mathrm{B}^{2} s\right\rangle /\left\langle\mathrm{B}^{2}\right\rangle\right)\right\rangle \\
& =-K^{-I} \mathrm{~T}\left(\mathrm{nv}{ }_{\theta} / \mathrm{B}_{\theta}\right)\left\langle\mathrm{B}^{2} \operatorname{lnn}\left(\operatorname{lnn}-\left\langle\mathrm{B}^{2} \ln n\right\rangle /\left\langle\mathrm{B}^{2}\right\rangle\right)\right\rangle \\
& =-k^{-1} \mathrm{~T}\left(n v_{\theta} / B_{\theta}\right)<B^{2}\left(\ell n n-<B^{2} \ell n n>/<B^{2}>\right)^{2}>
\end{aligned}
$$

This yields (20a).

\section{Inertial Corrections}

In evaluating (D3) and (D9), we only need $T$ to lowest order in $k$, and $n$ to lowest order in $1 / k$ li.e., only the "nondissipative" equilibrium quantities). However, note that (D3) and (D9) vanish to lowest order in $\left(\mathrm{v}_{\mathrm{p}} / \theta \mathrm{C}_{\mathrm{s}}\right)$. Thus inertial corrections to the equilibrium pressure are required. These are obtained from the parallel equation of motion (2a)

$$
(n M)^{-1}-\vec{B} \cdot \vec{\nabla} n\left(T_{e}+T_{i}\right)=-\vec{B} \vec{v}_{:} \vec{\nabla} \vec{v}
$$


Consider first the case where the electrons and ions are adiabatic and note that $T_{i} / T_{e}$ is a surface function. Using $s=(3 / 2) \ln T-\ln n$, the left hand side may be rewritten as

$$
\begin{gathered}
\vec{B} \cdot \vec{\nabla}\left(1+T_{i} / T_{e}\right)\left(T_{e} / M\right)+\left(I+T_{i} / T_{e}\right)\left(T T_{e} / M\right) \vec{B} \cdot \vec{\nabla} \ell n n \\
=(5 / 2)\left(1+T_{i} / T_{e}\right) \vec{B} \cdot \vec{\nabla}\left(T_{e} / M\right)
\end{gathered}
$$

Using the vector identity $\vec{v} \cdot \vec{\nabla} \vec{v}=\vec{\nabla}\left(v^{2} / 2\right)-\vec{v} \times(\vec{\nabla} \times \vec{v})$ and the relation $\vec{v} \times \vec{B}=\vec{\nabla} \phi$, we may now write (D10) as

$$
\begin{gathered}
(5 / 2)\left(1+T_{i} / T_{e}\right) \vec{B} \cdot \vec{\nabla}\left(T_{e} / M\right)=-\vec{B} \cdot \vec{V}\left(V^{2} / 2\right) \\
+\vec{\nabla} \cdot(\vec{\nabla} \phi x \vec{v})
\end{gathered}
$$

For our axisymetric geometry, we may integrate this once to give

$$
\left[(5 / 2)\left(1+T_{i} / T_{e}\right)\left(T_{e} / M\right)+\left(v^{2} / 2\right)+v_{z} v_{E} h / \theta\right]=\text { constant }
$$

Equations (10) are now used to rewrite the quantity $v^{2} / 2+v_{2} v_{E} h / \theta$ in terms of $v_{p}$ and $v_{E}$. This gives the desired result Eq. (19b), which represents a Bernoulli equation for the pressure. 
For the case where the electrons are isothermal and the ions adiabatic, the calculation proceeds similarily. The only difference is in the evaluation of $\mathrm{n}^{-1} \overrightarrow{\mathrm{B}} \cdot \vec{\nabla} \mathrm{p}$. By expanding in $\left(\mathrm{v}_{\mathrm{p}} / \theta \mathrm{C}_{\mathrm{s}}\right)$, the required integration may be performed to yield $(20 \mathrm{~b})$. 


\section{REFERENCES}

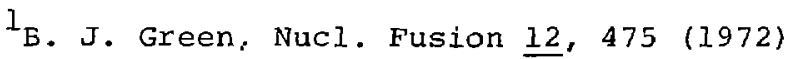

${ }^{2}$ T. E. Stringer, Phys. Rev. Lett. 22, 770 (1969)

3M. N. Rosenbluth, P. H. Rutherford, J. B. Taylor, E. A. Frieman, I. M. Kovxizhnikh, in Plasma Physics and Controlled Theomonuclear Fusion (International Atomic Energy Agency, Vienna, 1972), Vol. II, p. 479

${ }^{4}$ A. H. Boozar, Princeton Plasma Physics Laboratory Report EPRI-2 (1976)

${ }^{5}$ P. W. Chrisman, K. U. Von Hagenow and J. E. McCune, (to be published)

6. H. Stix, Phys. Fluids 16, 1260 (1973)

${ }^{7}$ R. D. Hazeltine, E. P. Lee and M. N. Rosenbluth, Phys. Rev. Lett. 25, 427 (1970)

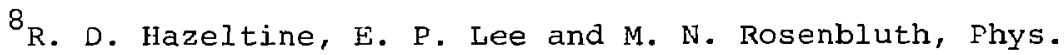
Fluids 14, 361 (1971)

${ }^{9}$ M. N. Rosenbluth and J. B. Taylor, Phys. Rev. Lett. $\underline{23}, 367$ (1969)

$10 \mathrm{~J}$. C. Hosea, F. C. Jobes, R. L. Hickok, and A. N. Dellis, Phys. Rev. Lett. 30, 839 (1973)

11 J. L. Terry, E. S. Marmar, K. I. Chen, and H. W. Moos, Phys. Rev. Lett. 39, 1515 (1977) 
12D. J. Sigmar, J. F. Clarke, R. V. Weidigh, and K. L. Vander Sluis, Phys. Rev. Lett. 33, 1376 (1974)

${ }^{13}$ s. I. Braginskii, in Reviews of Plasma Physics, edited by M. A. Leontovich (Consultants Bureau, New Yor', 1965), Vol. T, p. 214

${ }^{14}$ R. C. Grimm and J. I. Johnson, P1asma Phys. 14, 615 (1972). These authors were the first to employ the correct form for $\stackrel{\leftrightarrow}{\Pi}$, previously given expressions having been in error. ${ }^{15}$ E. K. Maschke, Plasma Phys. 14, 141 (1972)

${ }^{16} \mathrm{~A}$. A. Ware has pointed out to us the importance of these terms for flows of the order of the diamagnetic speed.

17 A. A. Galeev, JEPP Lett. 10,225 (1970)

${ }^{18}$ H. Grad and J. Hogan, Phys. Rev. Lett. 24, 1337 (1970) 


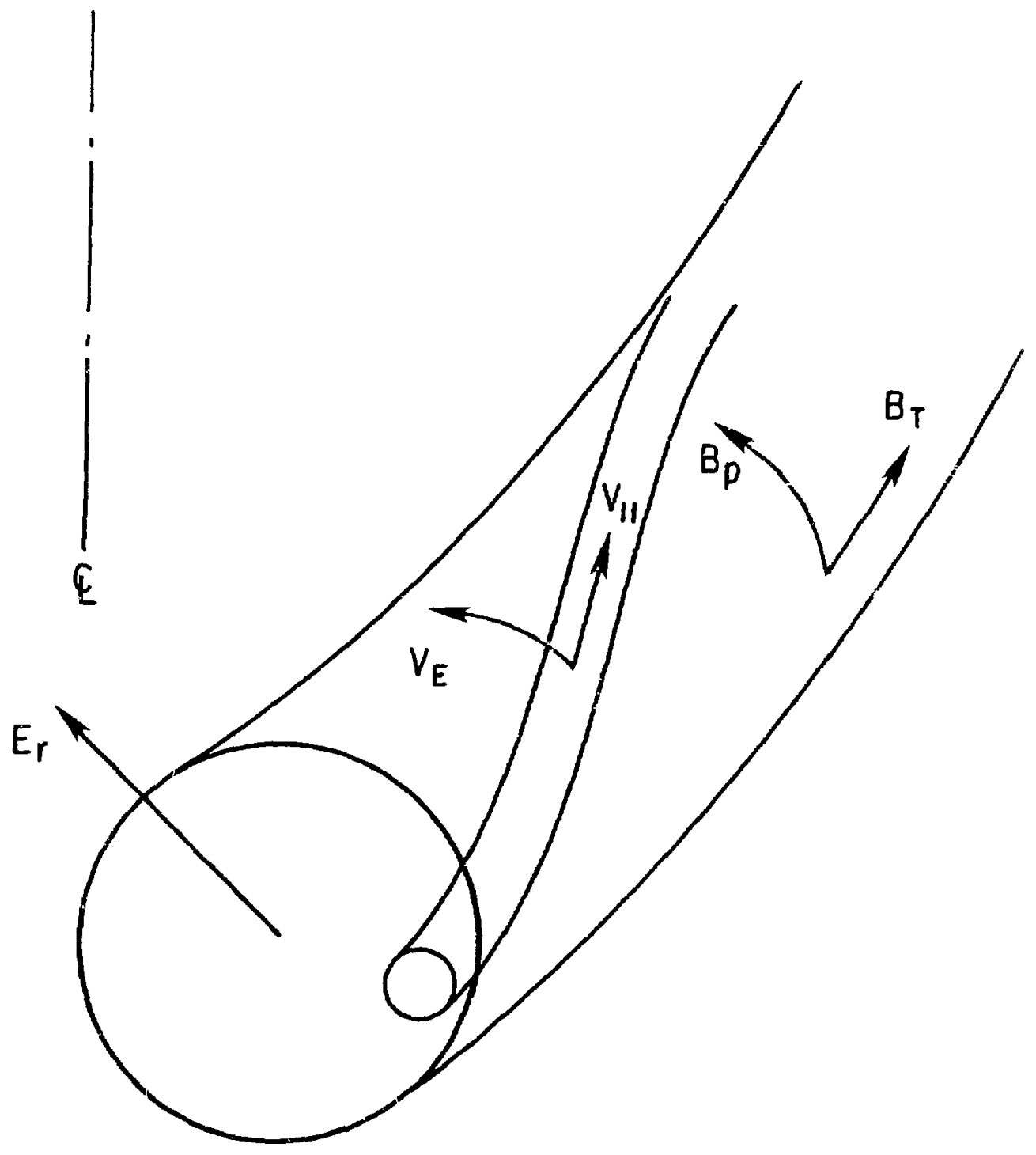

782033

Fig. I. Parallel flows superimposed on perperdicular flux tube rotation in a tokamak. 


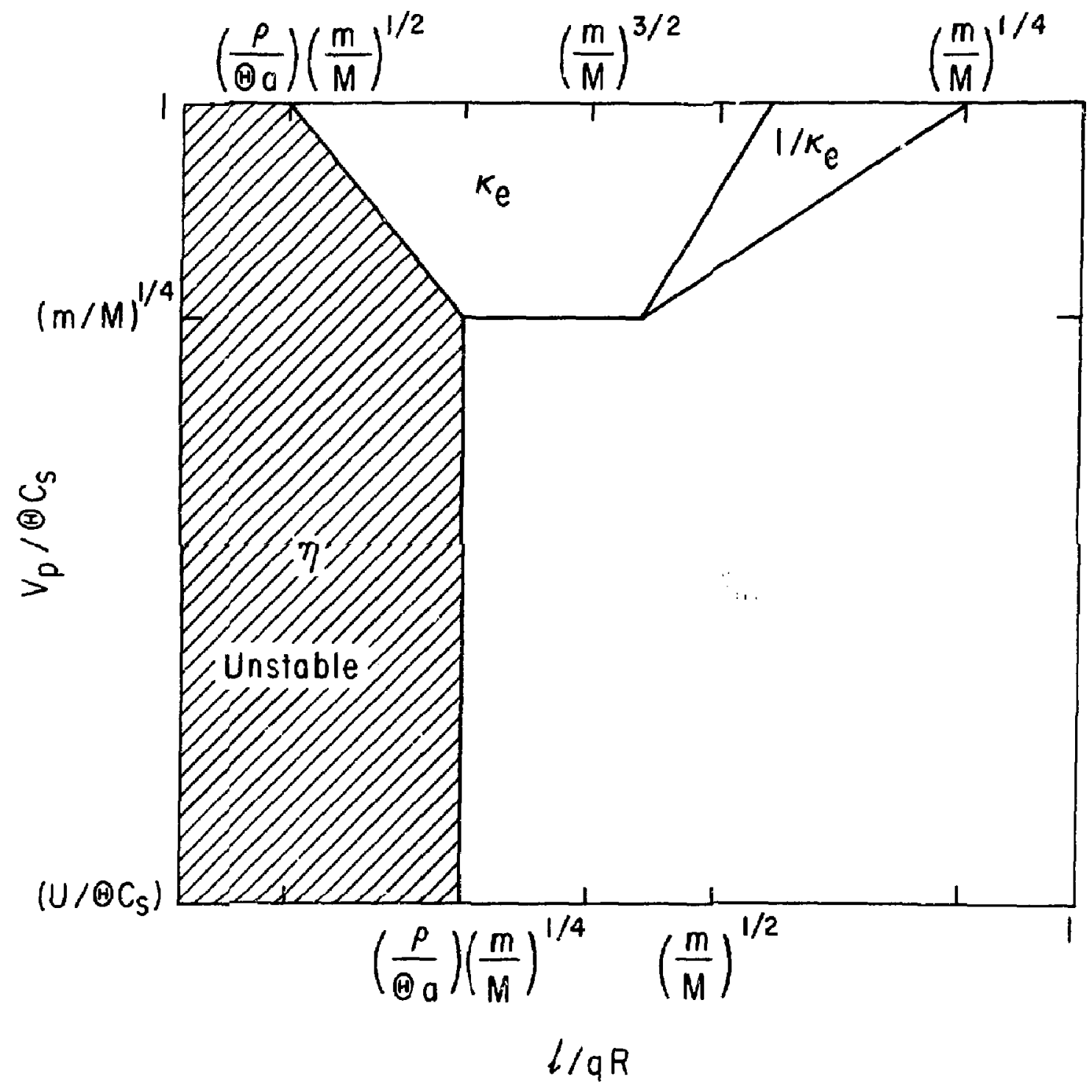

782034

Fig. 2. Schematic log-log parameter space plot demarcating the dominant dissipative processes which affect the rate of change of poloidal rotation. The plasma is unstable to poloidal rotation in the shaded region. 


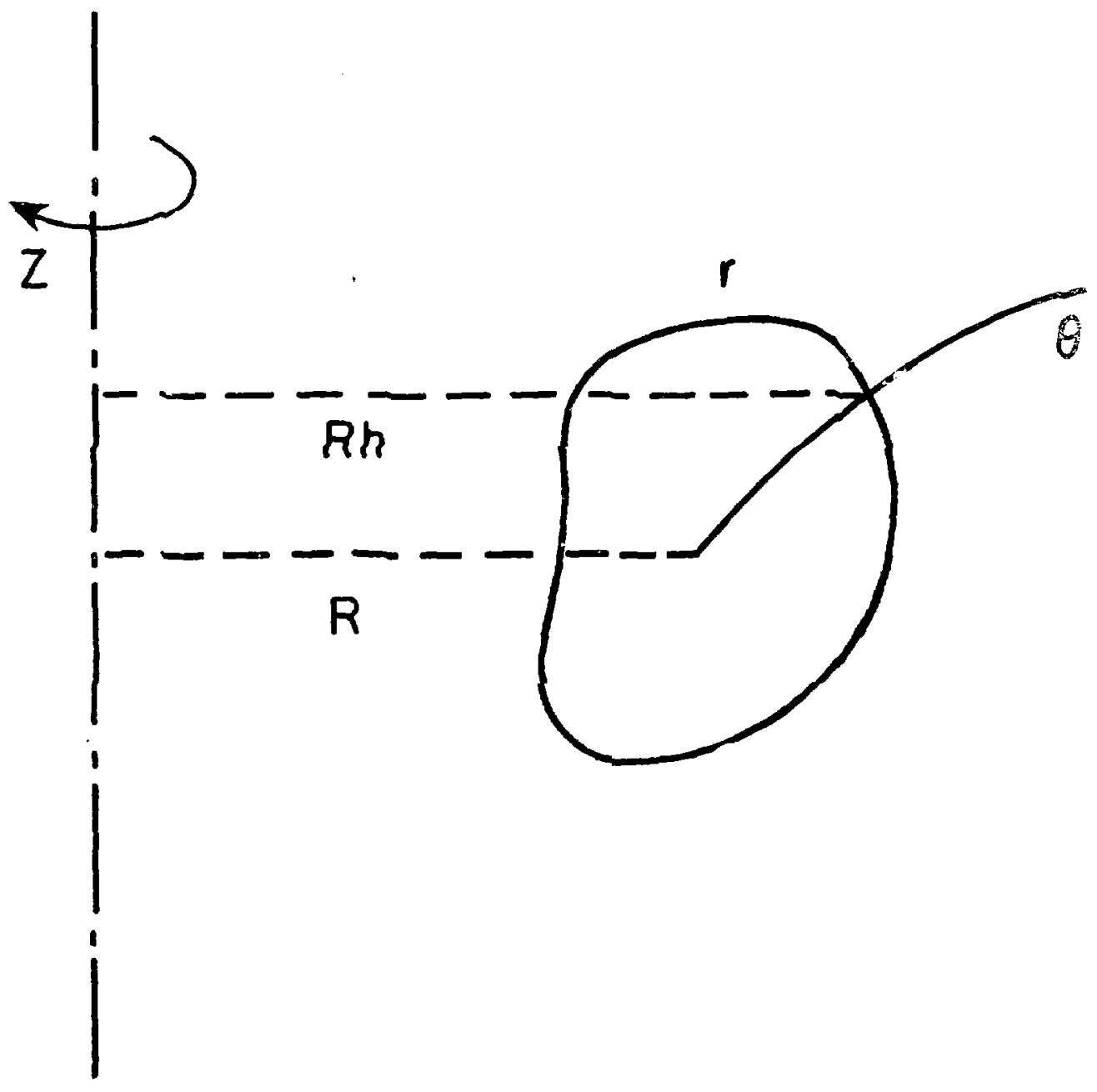

Fig. 3. The coordinate system. 


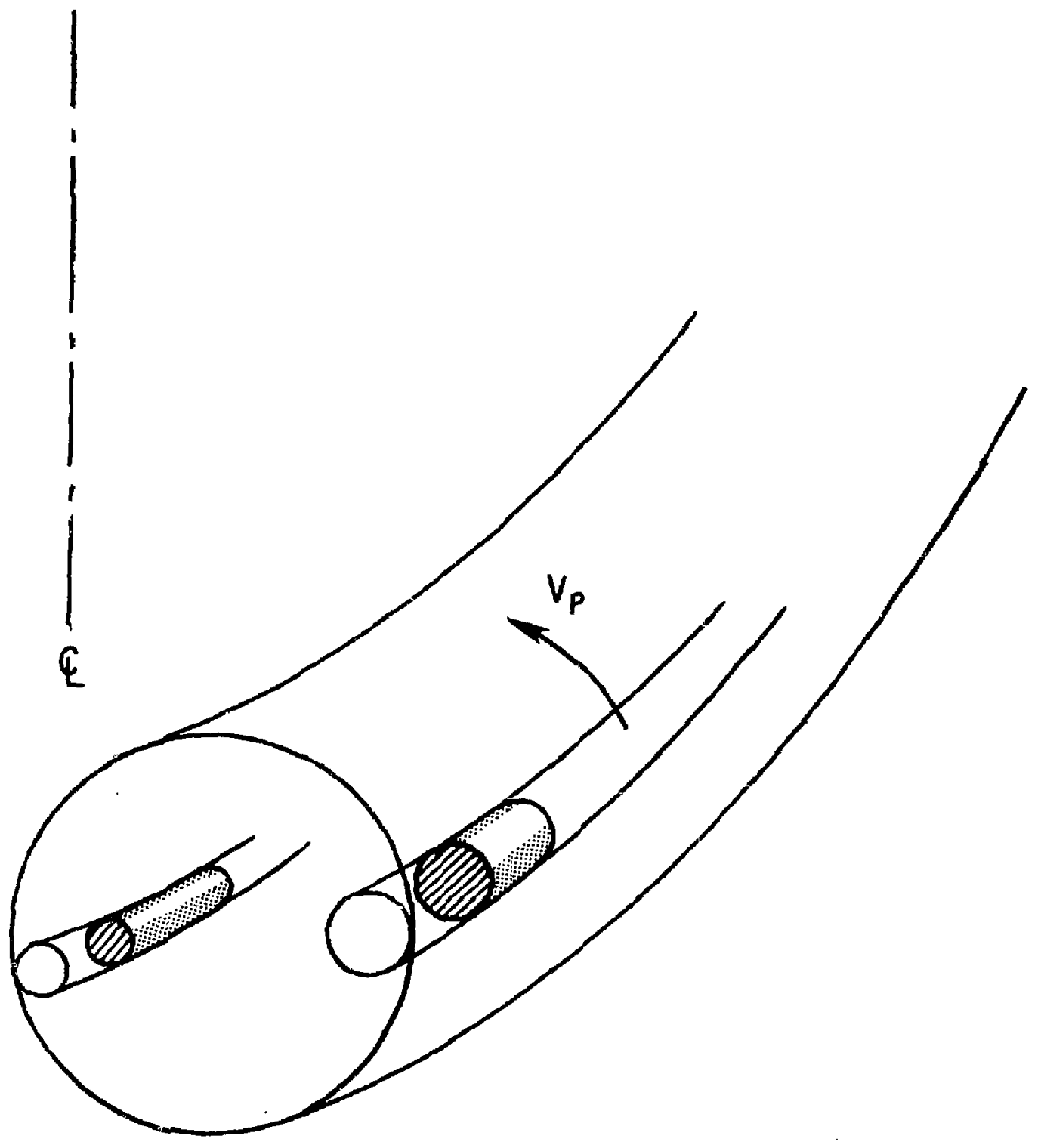

782032

Fig. 4. Sketch depicting the alternate parallel distention and perpendicular constriction of a fluid element in a rotating flux tube. 